\title{
http://bjas.journals.ekb.eg \\ Rule of Mac-2 Binding Protein Glycan Isomer in Assessment of Liver Fibrosis in Children with Chronic Liver Disease
}

\author{
S.A.El-Gendy ${ }^{1}$, O.G.Behairy ${ }^{1}$, A.I.Mansour ${ }^{2}$, O.S.El-Shimi ${ }^{1}$ and D.Y.Ibrahim ${ }^{1}$ \\ ${ }^{1}$ Pediatrics Dept., Faculty of Medicine, Benha Univ., Benha, Egypt \\ ${ }^{2}$ Clinical and Chemical Pathology Dept., Faculty of Medicine, Benha Univ., Benha, Egypt
}

E-Mail:Dalia@gmail.com

\begin{abstract}
Liver biopsy is the most accurate examination method to evaluate liver fibrosis stage but is invasive. Non-invasive biomarkers of disease progression are therefore needed. Assess the role of serum Mac-2 Binding Protein Glycan Isomer levels in diagnosis of liver fibrosis in children with chronic liver disease. This examine might have been an instance controlled ponder that might have been directed with respect to an aggregate number from claiming 160 Youngsters about both sexes. 100 know youngsters (case group) gathered starting with outpatient pediatric hepatology facility of Benha school doctor's facilities. Sixty Obviously sound kids (control group) matched the tolerant gathering done period Furthermore sex. Every one kids subjected on full historical backdrop taking, physical examination and research center Investigations, liver biopsy Also serum M2BPGI level Eventually Tom's perusing elisa. There might have been measurable noteworthy Contrast between contemplated gatherings in regards serum M2BPGI Similarly as it might have been raised over constant liver infection gathering. There were measurable noteworthy contrasts the middle of serum level for M2BPGI What's more fibrosis index, histological action list Similarly as it expands with build fibrosis list Furthermore hepatitis action list. The best cut off worth about serum M2BPGI might have been $1.06 \mathrm{ng} / \mathrm{ml}$ for identification from claiming promptly fibrosis phase for affectability $87.1 \%$, specificity half What's more range under those bend may be 0.823 same time the best cut off serum M2BPGI for identification of propelled fibrosis might have been $5.55 \mathrm{ng} / \mathrm{ml}$ with affectability $97.8 \%$, specificity 92.7 What's more territory under those bend is 0.987 . M2BPGI might a chance to be An novel non-invasive biomarker for liver fibrosis carried out with respect to egyptian know youngsters with liver infection.
\end{abstract}

Keywords: Chronic liver disease, Liver fibrosis, M2BPGI.

\section{Introduction}

Constant liver infection (CLD) may be a genuine wellbeing concern around the world. Without correct management, CLD progresses should liver fibrosis Also therefore prompts liver cirrhosis, which expands horribleness Also mortal sin brought on Eventually Tom's perusing portal hypertension, hepatic insufficiency, and the improvemen from claiming hepatocellular carcinoma (HCC) [1].

Pediatric liver infections contain a totally mixed bag from claiming disorders, including infections, developmental abnormalities, genetic, Also metabolic issue that Eventually bring about progressive alterations for structure from claiming liver and might end in cirque What's more its results [2].

Constant hepatitis may be regularly asymptomatic early Previously, its span Furthermore will be distinguished main Eventually Tom's perusing liver lab investigations for screening purposes or on assess nonspecific manifestations Yet patients could create protected manifestations comparable to intense hepatitis for example, nausea, vomiting, abdominal torment Also jaundice. It camwood prompt fibrosis through liver aggravation and increments those danger of liver cirque and hepatocellular carcinoma [3].

Analysis about Ceaseless hepatitis may be committed on the premise for some or constantly on of the following: a patient's indications Also symptoms, medicinal history, blood tests, imaging and liver biopsy [4].

In spite of the fact that liver biopsy is acknowledged the gold standard for stratifying hepatic fibrosis, it is invasive, its symptomatic worth will be constrained by inspecting error, Also histologic interpretations might differ. Likewise difficulties about $\mathrm{lb}$ are as a rule minor difficulties will incorporate pain, subcapsular dying that doesn't require transfusion alternately prolonged hospitalization, infection, minor bile spill or haemobilia, Furthermore arteriovenous fistula. Major difficulties incorporate bleeding, including haemobilia that obliges transfusion, surgery, alternately emergency unit administration; pneumothorax or haemothorax; Also passing. Therefore, noninvasive tests on assess liver fibrosis bring emerged, which incorporate elastographic techniques, serum biomarkers for example, hyaluronic acids, kind iv collagen, and kind iii procollagen-Npeptide and in addition surrogate markers (e. G. , those AST-to-platelet proportion list (APRI), and the FIB-4 index [5].

Mac-2 tying protein glycosylation isomer is a discharged glycoprotein that is introduce in the extracellular grid of a few tissues, otherwise called wisteria floribunda agglutinin-positive Mac2 tying protein with glycosylated isomer (WFA-M2BP) [6]. It may be a glycoprotein discharged from a number Mobile types, including hepatocytes, What's more its principle characteristic will be oligomerization to expansive ring structures that would possibly secured for sialylated $\mathrm{N}$-glycans. Clinched alongside constant liver disease, glycans are alluded will Likewise the face from claiming units that reflect liver fibrosis instead of liver inflammation, Furthermore progressions in the Nglycosylation of M2BP would reflected Toward those 
degree from claiming liver infection. Serum WFA+M2BP levels have been demonstrated will build with the progression from claiming liver fibrosis. Moreover, serum WFA+-M2BP levels need the limit should survey liver fibrosis without constantly influenced Eventually Tom's perusing tissue aggravation Also variances over alt values [7].

\section{Patients and methods}

This cross sectional instance regulated contemplate might have been directed ahead an aggregate amount about 160 kids from claiming both sex. 100 Youngsters gathered starting with participation from claiming outpatient pediatric hepatology facility from claiming Benha school healing facilities then afterward educated assent. 60 clearly solid kids were chosen starting with general populace Throughout those period starting with june 2018 with Ipril 2019. Lab worth of effort might have been directed for clinical pathology department, Benha school healing centers.

Group I (case group): included 100 children with chronic liver disease included (46 females/ 54 males) their mean age \pm SD $7.9 \pm 3.2$ years ranged from 2 to 17 years.

Group II (control group): included 60 children apparently healthy matching the case group for age and sex included (29females/ 31 males) their mean age \pm SD $7 \pm 2.8$ years ranged from 2 to 17 years.

\section{Inclusion criteria}

All children less than 18 years old with chronic liver disease of different etiology (e.g viral hepatitis, autoimmune hepatitis and cholestasis included extra hepatic biliary atresia and giant cell hepatitis), metabolic liver disease represent included (glycogen storage disease, Niemen pick disease,Wilson disease).

\section{Exclusion criteria}

Children with chronic liver diseases with comorbidities like renal diseases or heart diseases.

\section{Informed consent}

Informed consent was obtained from the parents after had been informed about all study procedures and their cosent was obtained prior to children enrollment in the study.

\section{Ethical approval}

This study was approved by the ethical committee of the Faculty of Medicine, Benha University.

\subsection{Methods}

All enrolled children were subjected to the following:

\section{A) Careful history taking \\ Personal history}

Name, age, sex, residence and social levels.

Complaint and history of present illness with stress on
-Jaundice, fatigue and acute hepatic like illness

-Consciousness level

-Fever

-Abdominal pain

-Melena and other bleeding: hematuria, epistaxis or bleeding gums

Past history with stress on

-Blood transfusion

-History of hepatitis B vaccine

-History of intake of hepatotoxic drugs

-Other autoimmune diseases

\section{Family history including}

-Parental consanguinity

-Similar condition in the family

-History of autoimmune disease in his/ her first degree relatives.

\section{B) Clinical examination}

\section{General examination}

-Level of consciousness and complexion (pallor, jaundice and cyanosis)

-Vital signs (heart rate, respiratory rate, blood pressure and temperature)

-Anthropometric measurements including: weight and height were recorded. Body mass index (BMI) calculated as $\mathrm{kg} / \mathrm{m} 2$. Weight was measured in $\mathrm{kg}$ using an electronic digital scale and its accuracy was periodically verified using reference weights. Length was measured in $\mathrm{cm}$. children were measured on scale with height gauges, the subject standing with back against the gauge and feet on the weighting platform. Patients height and weight for age percentiles were checked according WHO growth charts 2006 and 2007 (8\&9).

-Clubbing, purpura, scratch marks, palmar erythema, spider nevi and fetor hepaticas

-Lower limb edema.

\section{Abdominal examination}

1-Inspection: for abdominal wall veins, abdominal size and contour, divarication of recti, subcostal angle width and localized swelling.

2-Palpation:

-Superfacial palpation: for tenderness and $\backslash$ or rigidity.

-Deep palpation: for the liver and spleen.

Liver: The lower border of the liver in the right mid clavicular line in $\mathrm{cm}$ and percuss the upper border to detect liver span then detect lower border of the liver in the midline in $\mathrm{cm}$. In addition, detect the edge, consistency, surface, tenderness and pulsations in liver.

Spleen: The size of the spleen below costal margin in $\mathrm{cm}$, edge, consistency, surface and tenderness.

3-Percussion:

- For the presence of ascites.

- The upper border of the liver. 


\section{C) Abdominal ultrasound}

For assessment of liver span, texture, spleen span and presence of ascites.

\section{D) Laboratory investigations}

Sampling: Venous blood was drawn Seven ml venous blood was collected from each subject by clean venipuncture using disposable plastic syringe and divided as follows:

- $1 \mathrm{ml}$ on $150 \mu \mathrm{L} \mathrm{K} \mathrm{K}_{2}$-EDTA for CBC

- $2.25 \mathrm{ml}$ on $250 \mu \mathrm{L} \mathrm{ml} \mathrm{sodium-citrate} \mathrm{for} \mathrm{coagulation}$ studies

- $3 \mathrm{ml}$ on plain tube that was allowed to clot at room temperature for 30 min then serum was separated by centrifugation on $1262 \mathrm{~g}$ for $10 \mathrm{~min}$ for biochemical and serological testing.

The rest of serum was stored at $-20^{\circ} \mathrm{C}$ for furtherM2BPGI testing.

Biochemical and hematological investigations:

-Complete blood count (CBC): was done by automated hematology analyzer (CELL-DYN Emerald, Germany) to determine hemoglobin level ( $\mathrm{Hb})$, total leucocytic count (TLC) and platelets count (PLT)

-Prothrombin time (PT), concentration, INR and activated partial thromboplastin time (aPTT) (STA Compact Max, STAGO, France)

-Liver function tests: included serum total and indirect bilirubin, liver enzymes (ALT, AST, GGT, and alkaline phosphatase), serum total protein and albumin levels (BioSystems A25, Spain).

Table (1) Modified HAI Grading: Necroinflammatory Score.
All biochemical tests were performed as a routine laboratory investigation by appropriate instruments and kits. The upper limits of normal (ULN) for ALT and AST were established as $40 \mathrm{IU} / \mathrm{L}$. Viral markers, auto-antibodies and
Immunoglobulin G(IgG):

-Hepatitis b surface antigen (HBsAg), anti-HBs antibodies, anti-HB center Abdominal muscle (Igm and $\mathrm{IgG}$ ), anti-HCV Abdominal muscle What's more antiHAV Abdominal muscle (IgM What's more IgG) were tried Eventually Tom's perusing catalyst interfaced immunosorbent test (ELISA) unit (Biokit, Spain). Patients suspected on need immune system hepatitis were tried for serum autoantibodies including: antinuclear antibodies (ANA), anti-smooth muscle immunizer (ASMA), liver kidney microsomal antibody- 1 (LKM1), Also anti- mitochondrial immunizer (AMA) Eventually Tom's perusing aberrant immunofluorescence technobabble (NOVA Lite, Inova Diagnostics, Spain). -Immunoglobulin g ( $\operatorname{IgG})$ : by spiral immunodiffusion utilizing (IgG-NLRID, RN004. 3 , tying site , birmingham, UK). E) Percutaneous liver biopsy: Ultrasound guided liver biopsy done for all patients using Menghini aspirarion needle (Hepafix Luer Lock Melsungen AG, 3409 Mel-Sungen, Germany). Adequate core containing at least 5 portal tracts. Biopsy specimen were fixed in formaline and embedded in paraffin. Five $\mathrm{cm}$ thick section were cut, mounted on glass slide and stained with hematoxyline and eosin to evaluate histological activity of hepatitis using Ishak scoring index Table $(1,2)$. Also stained with Mason-Trichrome to assess fibrosis stage.

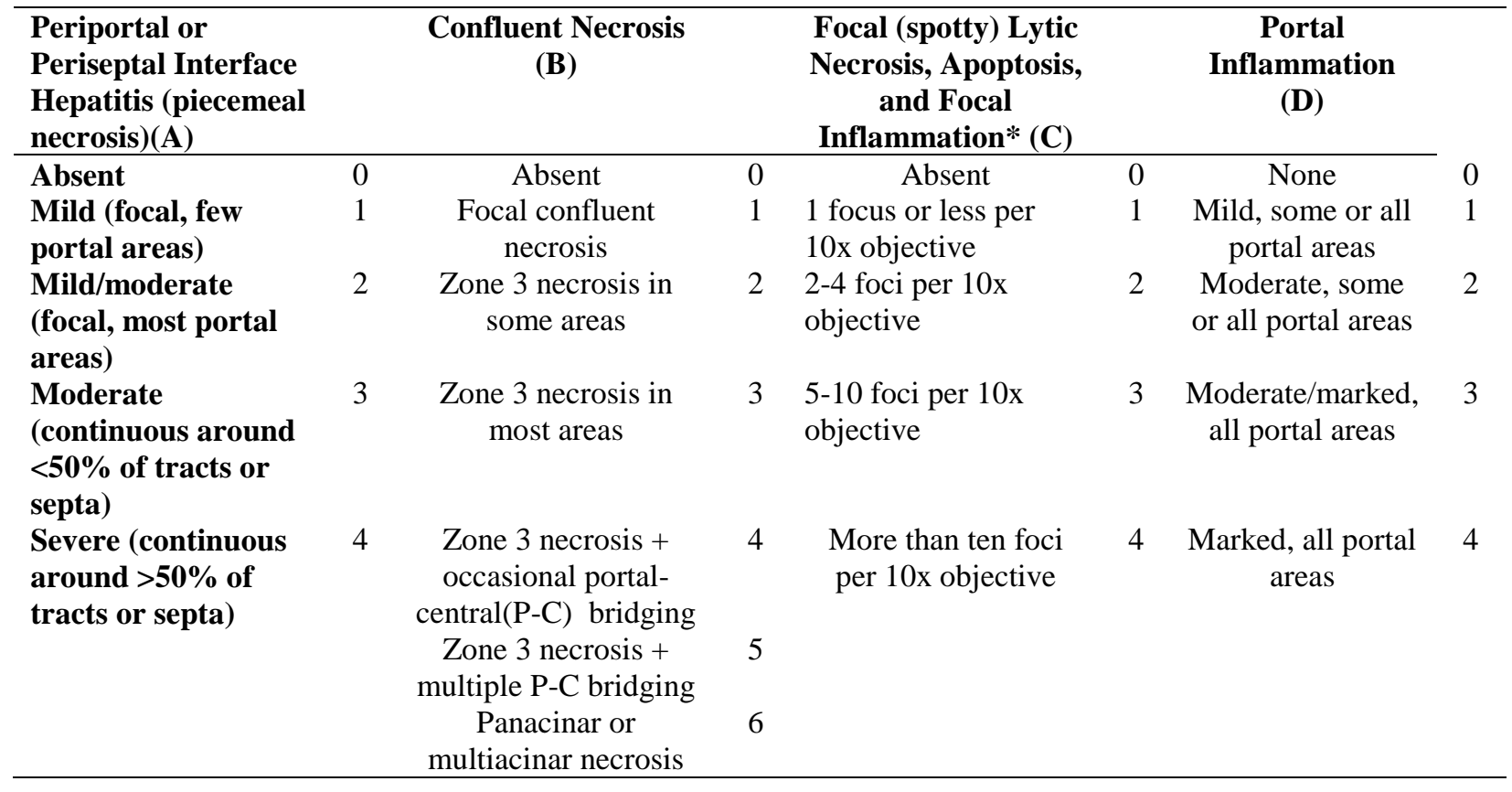

Total Modified HAI = /18. Total activity scores are defined as follows (1-3, minimal; mild 9-12; 13-18, sever) [10]. 
Table (2) Modified Staging: Architectural changes, fibrosis and cirrhosis.

\begin{tabular}{lc}
\hline \multicolumn{1}{c}{ Change } & Score \\
\hline No fibrosis & 0 \\
Fibrous expansion of some portal areas, with or without short fibrous septa & 1 \\
Fibrous expansion of most portal areas, with or without short fibrous septa & 2 \\
Fibrous expansion of most portal areas with occasional portal to portal (P-P) bridging & 3 \\
Fibrous expansion of portal areas with marked bridging [portal to portal (P-P) as well & 4 \\
as portal to central (P-C)] & 5 \\
Marked bridging (P-P and/or P-C) with occasional nodules (incomplete cirrhosis) & 6 \\
Cirrhosis, probable or definite & 5 \\
\hline
\end{tabular}

The disease severity scoring systems

For predicting prognosis of end-stage liver disease, many prognostic models were proposed as:

a) PELD score (pediatric end stage liver disease score): It is a disease severity scoring system for children under 12 years of age. This score is used for prioritizing allocation of liver transplants to predict survival. It is calculated according to the following formula :

PELD $=4.80 \times \operatorname{Ln}[$ serum bilirubin $(\mathrm{mg} / \mathrm{dl})]+18.57 \times$ Ln [INR] $-6.87 \times$ Ln [albumin $(\mathrm{g} / \mathrm{dl})]+4.36$ [11]. b) MELD score (model for end-stage liver disease score): It is a scoring system for assessing the severity of chronic liver disease. It was found to be useful in determining prognosis and prioritizing for receipt of a liver transplant. It is calculated according to the following formula [11]:

MELD $=3.78 \times$ Ln [serum bilirubin $(\mathrm{mg} / \mathrm{dl})]+11.2 \times$ $\mathrm{Ln}[\mathrm{INR}]+9.57 \times \ln [$ serum creatinine $(\mathrm{mg} / \mathrm{dl})]+6.43$.

C) Child-Pugh score Table (3)

Table (3) Child-Pugh score [12].

\begin{tabular}{lccc}
\hline Measure & 1 point & 2 points & 3 points \\
\hline Total bilirubin, $\boldsymbol{\mu m o l} / \mathbf{L}$ & $<34$ & $34-50(2-3)$ & $>50(>3)$ \\
(mg/dl) & $(<2)$ & & \\
$\begin{array}{l}\text { Serum albumin, g/dl } \\
\text { Prothrombin time, }\end{array}$ & $>3.5$ & $2.8-3.5$ & $>2.8$ \\
prolongation (s) & $<4.0$ & $4.0-6.0$ & $>6.0$ \\
Ascites & None & Mild or suppressed with & $\begin{array}{c}\text { Moderate to Severe or } \\
\text { refractory }\end{array}$ \\
Hepatic encephalopathy & None & medication & Grade III-IV \\
\hline
\end{tabular}

Child A: 5-6

Child B: 7-9

Child C: 10-15

Mac2 binding protein glycan isomer (M2BPGI) level Adouble-antibody sandwich ELISA (Enzyme Linked Immune Sorbent Assay) was used to detect serum level of Mac2 binding protein glycan isomer (M2BPGI) using Human (M2BPGI) ELISA Kit for research use only (Cat \#: SG-11567, China).

\section{Assay principle}

The kit is for the quantitative level of Human M2BPGI in the sample, adopt purified M2BPGI antibody to coat microtiter plate, make solid-phase antibody, then add M2BPGI to wells, Combine M2BPGI antibody with labeled HRP to form antibodyantigen-enzyme-antibody complex, after washing completely, add TMB substrate solution, TMB substrate becomes blue color at HRP enzyme-catalyzed, reaction is terminated by the addition of a stop solution and the color change is measured at a wavelength of $450 \mathrm{~nm}$. The concentration of M2BPGI in the samples is then determined by comparing the O.D. of the samples to the standard curve.

\section{- Standard curve (table (XIV))}

\begin{tabular}{lc}
\hline Standards concentration & O.D. (450 $\mathbf{~ m m})$ \\
\hline Standard (1): $\mathbf{1 . 2 5 n g / m l}$ & 0.044 \\
Standard (2): $\mathbf{2 . 5 n g / m l}$ & 0.105 \\
Standard (3): $\mathbf{5} \mathbf{~ n g / m l}$ & 0.242 \\
Standard (4): $\mathbf{1 0} \mathbf{~ n g / m l}$ & 0.511 \\
Standard (5): $\mathbf{1 5} \mathbf{~ n g / m l}$ & 0.781 \\
Standard (6): $\mathbf{2 2 . 5 n g / m l}$ & 1.189 \\
\hline
\end{tabular}




\section{Standard curve of M2BPGI}

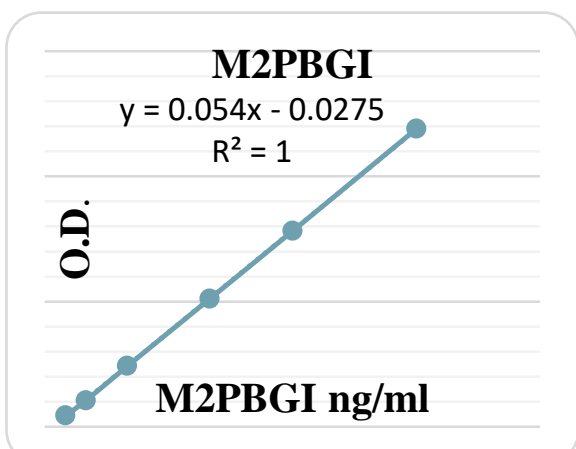

\subsection{Statistical methods}

Comes about were organized, tabulated Also statistically investigated utilizing SPSS product measurable workstation bundle versify 16 . Qualitative information were communicated as number Also rate. To quantitative data, those imply and standard deviation Also average were computed. Those distinction between methods might have been statistically broke down utilizing the scholar ( $\mathrm{t}$ ) test, Mann-Whitney test What's more anova test The point when might have been suitableness relationship examination might have been carried out utilizing Pearson(r) Also Spearman (rho) relationship when might have been suitableness. Collector driver trademark (ROC) bend might have been used to evaluate the execution from claiming M3BPGI Previously, analysis for fibrosis. Those acknowledged level about noteworthiness in this worth of effort might have been $0.05(\mathrm{p} \leq 0.05)$.

$\mathrm{P}$ value $>0.05$ is non-significant (NS)

$\mathrm{P}<0.05$ is significant $(\mathrm{S})$

$\mathrm{P} \leq 0.001$ is highly significant (HS)

\section{Result}

\section{Demographic characteristics}

There was no significant difference between both groups as regard to age, gender and consanguinity Table (4).

\section{Anthropometric measurements}

There was a statistical significant difference between chronic liver disease patients and control group regarding weight, weight/ centile, height and height/ centile, body mass index (BMI) and BMI/ centile as it was lower in chronic hepatitis group Table (5).
Research center parameters: there might have been a Factual huge distinction $(\mathrm{P}$ esteem $<0.01)$ between both aggregations in regards a few parameters. Hb level Also platelet number were easier Previously, Ceaseless liver infection aggregation over control group, same time PT, INR, AST, ALT, GGT, high mountain Furthermore bilirubin (total\& direct) were higher On constant liver infection gathering over control assembly. However, there might have been no measurable huge distinction Around Ceaseless liver infection gathering and control one assembly in regards TLC, egg whites.

M2BPGI: there might have been Factual huge Contrast the middle of mulled over aggregations viewing serum M2BPGI level Concerning illustration it might have been raised done incessant liver infection bunch.

There might have been Factual critical certain connection between serum level for M2BPGI Also outcomes about liver biobsy. Serum level from claiming M2BPGI expanded with expanded fibrosis index, hepatitis action list Also interface hepatitis. There were huge certain correlations the middle of M2BPGI, liver work test (GGT, AST, ALT, ALP, billirubin (total and direct)) and fibrosis score same time there might have been negative correspondence between M2BPGI, hb Furthermore platelets.

Those best cut off quality about serum M2BPGI might have been 1.06 identification for early fibrosis phase with affectability $87.1 \%$, specificity half What's more region under the bend may be 0.823 same time those best cut off serum M2BPGI to identification of moderate fibrosis might have been 5. 55 for affectability $97.8 \%$, specificity $92.7 \%$ and zone under the bend is 0.987 .

Table (4) Demographic characteristics of the studied groups.

\begin{tabular}{|c|c|c|c|c|c|}
\hline Variables & & $\begin{array}{l}\text { Hepatic group } \\
(\mathrm{n}=100)\end{array}$ & $\begin{array}{l}\text { Controls } \\
(n=60)\end{array}$ & Test & $P$ value \\
\hline \multirow[t]{2}{*}{ Age } & Mean \pm SD & $7.9 \pm 3.2$ & $7 \pm 2.8$ & $\mathrm{t}=1.8$ & 0.07 \\
\hline & Range & $2-17$ & $2-17$ & & \\
\hline \multirow[t]{2}{*}{ Gender } & Males $\quad \mathrm{n}(\%)$ & $54(54.0)$ & $31(51.7)$ & $\mathrm{X} 2=0.082$ & 0.775 \\
\hline & Females n (\%) & $46(46.0)$ & $29(48.3)$ & & \\
\hline Consanguinity & Positive $\mathrm{n}(\%)$ & $42(42.0)$ & $24(40.0)$ & $\mathrm{X} 2=0.062$ & 0.804 \\
\hline
\end{tabular}


Table (5) Anthropometric measurements between studied groups.

\begin{tabular}{|c|c|c|c|c|c|}
\hline variables & & $\begin{array}{r}\text { Hepatic group } \\
(\mathrm{n}=\mathbf{1 0 0}) \\
\end{array}$ & $\begin{array}{r}\text { Controls } \\
(\mathrm{n}=60) \\
\end{array}$ & Test & $P$ value \\
\hline Weight (kg) & $\begin{array}{c}\text { Mean } \pm \text { SD } \\
\text { Range }\end{array}$ & $\begin{array}{c}20.5 \pm 15.3 \\
3-50\end{array}$ & $\begin{array}{c}25.88 \pm 17.13 \\
3.5-67\end{array}$ & $t=2.04$ & $0.043^{*}$ \\
\hline Weight on centile & $\begin{array}{c}\text { Mean } \pm \text { SD } \\
\text { Range }\end{array}$ & $\begin{array}{l}28.2 \pm 18.9 \\
2-50\end{array}$ & $\begin{array}{c}47.6 \pm 15.3 \\
10-75\end{array}$ & $\mathrm{t}=7.11$ & $<0.001 * *$ \\
\hline Height (cm) & $\begin{array}{l}\text { Mean } \pm \text { SD } \\
\text { Range }\end{array}$ & $\begin{array}{c}102 \pm 32.7 \\
52-150\end{array}$ & $\begin{array}{c}115.3 \pm 35.4 \\
54-170\end{array}$ & $\mathrm{t}=2.41$ & 0.016 \\
\hline Height on centile & $\begin{array}{l}\text { Mean } \pm \text { SD } \\
\text { Range }\end{array}$ & $\begin{array}{l}29.14 \pm 19.2 \\
2-50\end{array}$ & $\begin{array}{c}47 \pm 18.7 \\
10-80\end{array}$ & $t=5.79$ & $<0.001$ \\
\hline BMI & $\begin{array}{l}\text { Mean } \pm \text { SD } \\
\text { Range }\end{array}$ & $\begin{array}{c}15.9 \pm 3.06 \\
10-22\end{array}$ & $\begin{array}{c}17.0 \pm 2.6 \\
11-25\end{array}$ & $\mathrm{t}=2.37$ & 0.019 \\
\hline BMI on centile & Mean \pm SD & $25.9 \pm 17.7$ & $44.13 \pm 21.3$ & $t=5.83$ & $<0.001$ \\
\hline
\end{tabular}

\section{Discussion}

In the present study, there might have been measurable critical distinction between constant liver infection patients and control aggregation in regards weight, weight/ centile, height, height/ centile, figure impostor list Furthermore body impostor index/ centile as it might have been bring down On Ceaseless liver infection gathering. This information run to understanding for [13] who examined 131 unending liver infection patients Furthermore discovered that the sum anthropometric estimations that incorporate weight, height, particular figure impostor index, mid upper arm circumduction Also triceps skinfold were fundamentally more level "around cases over controls because of poor nourishment intake, disturbances for absorption Furthermore absorption for dietary substances in the gastrointestinal tract Also impeded hepatic union from claiming vitality substrates and these abnormalities bit by bit influence their. Anthropometric parameters. Also [14] who evaluated weight, stature Furthermore form impostor list done youths contaminated for HCV previously, then following medication for ledipasvir/ sofosbuvir Also discovered that the patients needed poor weight, tallness What's more physique impostor list When medicine and there may be change then afterward medication. Additionally [15] who examined 12 tyke contaminated for $\mathrm{HCV}$ and found that the kids contaminated with $\mathrm{HCV}$ need low weight, tallness What's more physique impostor list because of hunger Furthermore Growth impediment.

In the current study, there might have been Factual noteworthy distinction the middle of unending liver infection patients What's more control assembly viewing $\mathrm{HB}$, platelet, Similarly as they were bring down clinched alongside unending liver infection bunch over control group, same time PT, INR, AST, ALT, GGT, high mountain What's more bilirubin (total\& direct) were higher On unending hepatitis one assembly over control assembly. However, there might have been no measurable huge Contrast "around unending liver infection gathering Also control aggregation viewing tlc Also egg whites. These effects were in understanding with [16] who discovered that there might have been rise for AST, ALT, GGT Also bilirubin At hb might have been low. Likewise [17] who concentrated on 771 tolerant with incessant liver infection for assessing the phase about fibrosis Furthermore discovered that there might have been rise for AST, ALT, inr What's more high mountain At platelets and egg whites were diminished with expanding fibrosis phase. [18] who concentrated on 144 sure anti-HCV youngster Also 168 anti-HCVnegative tyke and found that platelets were easier to sure anti-HCV kids over anti-HCV negative know youngsters However there might have been rise done AST, alt and bilirubin for sure anti-HCV Youngsters.

Alt Furthermore AST levels increment for those progression about liver infection as continuous liver damage increments their arrival starting with mitochondria What's more fibrosis declines this freedom [19].

In the current study, there might have been measurable huge distinction the middle of constant liver infection patients and control one assembly viewing serum M2BPGI as it might have been higher for constant hepatitis aggregation (mean \pm sd might have been $6.04 \pm 2$. 72). These outcomes were in understanding for 20) who mulled over 680 patiant with incessant hepatitis c infection Also 164 control tolerant discovered that M2BPGi level differed altogether between hepatitis $\mathrm{c}$ patients and sound controls. Around incessant hepatitis c patients, those average level from claiming serum M2BPGi might have been positively connected with fibrosis phase Likewise 0. $88(<\mathrm{F} 2), 1.70$ (F2/F3), Furthermore 5. 68 (F4) yet the average serum M2BPGi level might have been 0.38 clinched alongside sound controls. Also [21] who concentrated on serum MAC2 glycan tying protein isomer level ahead 64 patiant for billiary atresia Also discovered that serum MAC2 glycan tying protein isomer levels were essentially higher Previously, patients over On sound controls. For patients with BA, roc Investigation exhibits that serum M2BPGi need finer symptomatic capacity to recognize fibrosis of the local liver for evaluation F4 fibrosis over different accepted serum biomarkers for example, such that hyaluronic acid, P-III-P, kind iv collagen $7 \mathrm{~s}$, 
alternately APRI. Likewise [22] who concentrated on serum Wisteria floribunda agglutinin-positive Mac-2binding protein levels anticipate those vicinity about fibrotic nonalcoholic steatohepatitis (NASH) Furthermore nash cirque for from claiming 20 solid controls What's more 198 patients with biopsy-proven NAFLD discovered that WFA+ -M2BP level might have been fundamentally expanded in the fibrotic nash What's more nash cirque gatherings contrasted with solid controls.

In the introduce study, there were Factual critical contrasts between serum level of M2BPGI Also fibrosis list and histological action list as it increments for expansion fibrosis list Furthermore histological action list. These discoveries were in understanding for [23] who found that there were correlations between serum WFA+-M2BPGI level What's more necroinflammation evaluation What's more fibrosis stage Previously, patiant for $\mathrm{HBV}$ as there might have been a noteworthy expand done serum WFA+-M2BP level as stated by necroinflammation evaluating and fibrosis stage. Also [21] who found that over patients for billiary atresia, the average M2BPGI esteem over patients for F4 review fibrosis might have been altogether higher over over patients for F3 fibrosis [24] . found that On tolerant for $\mathrm{HCV}$, there might have been a essentially expanded serum M2BPGI levels with the progression about liver fibrosis.

In the present study, there might have been certain relationship the middle of serum M2BPGI What's more liver proteins (PT, PTT, INR, AST, ALT, GGT, high mountain Also bilirubin (total\& direct)), same time there might have been negative correspondence between serum M2BPGI and hemoglobin and platelet. At there might have been no Factual critical correspondence between serum M2BPGI Furthermore aggregate leucocytic number What's more egg whites. These effects were clinched alongside [25] who mulled over convenience of serum Wisteria floribunda agglutinin-positive Mac-2 tying protein Previously, know youngsters with elementary sclerosing cholangitis found that there might have been huge certain connection between serum level of M2BPGI Furthermore AST, ALT, GGT, aggregate serum billirubin, igigi. Additionally (26) who mulled over the part of serum M2BPGi levels ahead diagnosing noteworthy liver fibrosis Furthermore cirque Previously, approached patients with incessant hepatitis b infection contamination discovered that albumin, AST, ALT, PLT and afp associated essentially for serum M2BPGi levels likewise [27] who mulled over serum wisteria floribunda agglutinin-positive Mac-2 tying protein level Similarly as An symptomatic marker for hepatitis $b$ infection found that there might have been An certain correspondence between serum WFA+-M2BP Furthermore AST, ALT, alpha fetoprotein (AFP). A negative correspondence might have been discovered between serum WFA+-M2BP levels Also platelet checks. There might have been no correspondence the middle of serum WFA+-M2BP levels Also different parameters (age, sex, aggregate bilirubin and albumin).

M2BPGi might have been discharged starting with actuated hepatic stellate phones (HSCs), which Additionally actuated those occupant macrophages, Kupffer phones. Those actuated macrophages proportionally actuated the HSCs, which prompted further liver fibrosis. Therefore, M2BPGi level rise demonstrates the actuation about HSCs, which may be the fibrogenic status Be that not those fibrotic status itself [28].

In the present study, the best cut off esteem about serum M2BPGI might have been 1. 06 for identification of early fibrosis phase for affectability $87.1 \%$, specificity half territory under those bend may be 0.823 same time those best cut off serum M2BPGI to identification about moderate fibrosis might have been 5.55 for affectability $97.8 \%$, specificity $92.7 \%$ Furthermore region under those bend may be 0.987 . These discoveries were in understanding with [29] Who mulled over Wisteria floribunda agglutininpositive Mac-2-binding protein in the prediction from claiming illness seriousness in Ceaseless hepatitis b patients discovered that the ideal cutoff quality might have been for F1 might have been 0.960 , yielding the affectability from claiming 61. 2\%, specificity of 76 . $2 \%$, the ideal cutoff esteem might have been 1.345 for F2, yielding the affectability of $65.9 \%$, specificity from claiming $80.6 \%$ and the ideal cutoff qualities for F3 Furthermore F4 were 1. 535, What's more 1. 665awith affectability 67. 7, 69. 2 and specificity 79. 8, 76. 1 individually. Likewise [30] who found that M2BPGi values went starting with 0.3 on 6.9 cut off list (COI) (median 1. 6). The degree about liver fibrosis differed for M2BPGi level. For foreseeing cirque (F4) Also propelled liver fibrosis ( $\geq$ F3), M2BPGi needed higher ranges under the bend (AUCs; 0. 93, respectively) with cut off COIs from claiming 1.84 and 1. 67, individually. Also [26] who contemplated part of serum M2BPGi levels once diagnosing critical liver fibrosis Also cirque done treated patients with Ceaseless hepatitis $\mathrm{b}$ infection spoiling discovered that the average M2BPGi qualities to F0/F1/F2, F3 Also F4 progressively expanded for that's only the tip of the iceberg propelled phases from claiming liver fibrosis: the cut-off qualities about 0. 605 What's more 0. 615 COI, the AUROCs were 0. 754 What's more 0.799 to $\geq$ F3 Also F4, affectability 62. 5\%, 73. 6\% and specificity $79.4 \%, 77 \%$, individually.

\section{Conclusion}

Serum M2BPGI may be agood non- invasive biomarker for liver fibrosis in Egyption children with chronic liver disease.

\section{References}

[1] R. N. karanjia, M. M. Crossey, 1. J. Cox, Hepatic steatosis and fibrosis: non-invasive assessment. World J Gastroenterol,Vol. 22, PP.9880-97, 2016. 
[2] S. Narayan, M. Gautam, H. Dipankar, Spectrum of hepatobiliary disease among children in Northeast India. J Dent Med Sci, Vol. 16(7), PP.50-4, 2017.

[3] Y. Nagaoki, M. Imamura, H. Aikata, The risks of hepatocellular carcinoma development after HCV eradication are similar between patients treated with peg-interferon plus ribavirin and direct acting antiviral therapy. PLoS One, Vol. 12, PP.e0182710, 2017.

[4] H. Friedman, S. Lawrence, N..Papadakis, Liver, Biliary Tract, \& Pancreas Disorders. In Current Medical Diagnosis \& Treatment. McGraw Hill,Vol. Chapter 16, pp.55e ISBN 0071845097, 2015.

[5] Y. Lurie, M. Webb, R. Cytter-Kuint, Non-invasive diagnosis of liver fibrosis and cirrhosis. World $\mathrm{J}$ Gastroenterol, Vol. 21, PP.11567-83, 2015.

[6] Y. Bekki, H. Yoshizumi, S. Shimoda, Hepatic stellate cells secrete WFA? M2BP. Its role in biological interactions with Kupffer cells. J Gastroenterol Hepatol,Vol.32, PP.1387-1393, 2015.

[7] K. Yamasaki, M. Tateyama, H. Tsujikaw, Elevated serum levels of WFA -M2BP predict the development of hepatocellular carcinoma in hepatitis C patients. Hepatology,Vol. 7, PP.244269, 2017.

[8] W. H. O., WHO Child Growth Standards: Length/Height-for-Age, Weight-for-Age, Weightfor-Length, Weight-for-Height and Body Mass Index-for-Age: Methods and Development. Geneva, Switzerland: World Health Organization, Vol. 20,pp.230-410, 2006.

[9] W. H. O.,WHO Child Growth Standards: Methods and Development. Head circumference-for-age, arm circumference-for-age, triceps skinfold-for-age and sub-scapular skinfold for age. Geneva, World Health Organization, Vol. 20,pp.230-120,2007.

[10] K. Ishak, A. Baptista , 1. Bianchi, Histological grading and staging of chronic hepatitis. J Hepatol,Vol.22, PP.696-699, 1995.

[11]R. B. Freeman, R. H. Wiesner , R. J. Roberts,Improving liver allocation: MELD and PELD. Am J Transplant, Vol. 4(9), pp.114-31, 2004.

[12] T. I. Huo , H. C. Lin,J. C. Wu ,Proposal of a modified Child Turcotte- Pugh scoring system and comparison with the model for end-stage liver disease for outcome prediction in patients with cirrhosis. Liver Transpl,Vol.12, pp.65-71, 2004.

[13] G. Nunes, G. S. Santos, R. Barbosa, outcome and nutritional assessment of chronic liver disease patients using anthropometry and subjective global assessments. Arq Gastroenterol, Vol.s54(3), PP.225-231, 2007.

[14]Z.M.Younossi, M. Stepanova S. Balistreri, Health-related quality of life in adolescent patients with hepatitis $\mathrm{C}$ genotype 1 treated with Sofosbuvir and Ledipasvir. J Pediatr Gastroenterol Nutr, Vol. 66, PP.112-116,2014
[15] K. F. Murray, C. Hepatitis, virus infection in children. Gastroenterol Hepatol (N Y), Vol. 13(3)PP.184-187, 2017.

[16] Abou-Taleb, R.h.Ahmed, A. Elhennawy,Pediatric chronic liver diseases: A clinico pathological study from a Tertiary Care Center. Int J pediatr, Vol. 7(4) , PP.Serial no 64, 2019.

[17]Z.Ahmed, M. Ahmed, S. Walayat, Liver function tests in identifying patients with liver disease. Clin Exp Gastroenterol,Vol.11, PP.301-307, 2018.

[18] M. H. Tsai, K. H. Lin, K. T. Lin, Predictors for early identification of hepatitis $\mathrm{C}$ virus Infection. Biomed Res Int,Vol.25,pp.429290, 2015.

[19]B. Nalpas, A. Vassault, A. Le Guillou, Serum activity of mitochondrial aspartate aminotransferase: A sensitive marker of alcoholism with or without alcoholic hepatitis. Hepatology, Vol. 4, PP.893-986, 1984.

[20] X. U. Hongqin, w. Kong , L. Liu , Accuracy of M2BPGi, compared with Fibro Scan, in analysis of liver fibrosis in patients with hepatitis C. BMC Gastroenterology, Vol. 17, PP.62, 2017.

[21] U. Yamada, Y. Sanada,M. Tashiro, Serum Mac-2 binding protein glycosylation isomer predicts grade F4 liver fibrosis in patients with biliary atresia. J Gastroenterol, Vol. 52, PP.245-252, 2017.

[22] N. Alkhouri , C. Johnson, 1. Adams, Wisteria floribunda agglutinin-positive Mac-2-binding protein levels predict the presence of fibrotic nonalcoholic steatohepatitis (NASH) and NASH cirrhosis. PLoS One, Vol. 13(8), PP.83-92, 2017.

[23] N. Alkhouri, C. Johnson, 1. Adams Serum Wisteria floribunda agglutinin-positive Mac-2-binding protein levels predict the presence of fibrotic nonalcoholic steatohepatitis (NASH) and NASH cirrhosis. PLoS One, Vol. 13(8), PP.8392, 2018.

[24] C. L. Huang , C. F. Huang, M. L. Yeh , Serum Wisteria floribunda agglutinin- positive Mac-2binding protein expression predicts disease severity in chronic hepatitis C patients. Journal of Medical Sciences, Vol. 33, PP.399, 2018.

[25] S. Umetsu, A. nui ,T. Sogo ,Usefulness of serum Wisteria floribunda agglutinin-positive Mac-2 binding protein in children with primary sclerosing cholangitis. Hepatology, Vol. 48, PP.355-363, 2018.

[26] L. K. Mak, D. J. Wong, Cheung K. S. Role of serum M2BPGi levels on diagnosing significant liver fibrosis and cirrhosis in treated patients with chronic hepatitis B virus infection. Clin Transl Gastroenterol, Vol.9(6), PP.163-170, 2018.

[27] N. Chuaypen K. Chittmittraprap Pinjaroen, B. Sirichindakul Serum Wisteria floribunda agglutinin-positive Mac-2 binding protein level as a diagnostic marker of hepatitis $\mathrm{B}$ virus related hepatocellular carcinoma. Hepatology Research, Vol. 48, pp872-881, 2019.

[28] K. Shirabe, Y. Bekki, D. Gantumur, Mac-2 binding protein glycan isomer (M2BPGi) is a new serum 
biomarker for assessing liver fibrosis: more than a biomarker of liver fibrosis. J Gastroenterol. 2018;53: 819-26.

[29]H.Yeh , C. F. Huang,D. Huang, Wisteria floribunda agglutinin-positive Mac2binding protein in the prediction of disease severity in chronic hepatitis B patients. PLoS ONE, Vol. 14(8), PP.1826,2014.
[30]Y. Eso, Takai A, K. Taura , Takahashi K, Association of Mac-2-binding protein glycosylation isomer level with nutritional status in chronic liver disease. Journal of Gastroenterology and Hepatology, Vol. 33, PP.li1649-1658, 2018. 